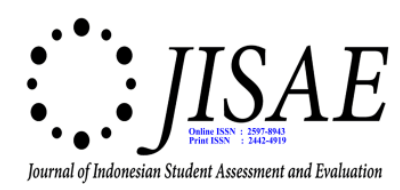

\title{
COMPUTER AIDED INSTRUCTIONAL MATERIALS IN TEACHING COOKERY IN GRADE 10: IT'S EFFECTIVENESS
}

\author{
Sharon T. Nicolas \\ Gen. Ricardo G. Papa Sr. Memorial High School \\ Gen. Luna St., Ususan, Taguig City \\ Email Address: sharonnicolas78@yahoo.com
}

\begin{abstract}
Today's world is known as the globalization era and is marked by rapid and complex changes both in social and values and learning structures. As such, it is natural for the education sector to review, update and orient their programs to the paradigm of these developments because education is crucial to global competitiveness and total human development. The main purpose/aim of this research is to determine the effectiveness of computer aided instructional materials in teaching cookery to grade 10 students. In this study, experimental method of research was used, the pretest and posttest design. The control group and the experimental group were both given a pretest, then exposed to different teaching methods. The experimental group was exposed to computer aided instructional materials while the control group was taught using the traditional approach. Both groups were given a posttest.The researcher used Mean, Percentage and T-test for the statistical treatment. Comparing the results of the pretest and posttest in the control and experimental group, it shows thatstudents who used computer aided instructional materials performed well in class. The result of the experimental group increases in the post test. Therefore, computer aided instruction enhances the learning abilities of the students.
\end{abstract}

Keywords: globalization era, paradigm, global competitiveness, complex

The general purpose of this study is to identify the effectiveness of Computer Aided Instructional Materials in Teaching Cookery in Grade 10.

In recent years, discussion has increased around the apparent decline of literacy levels. Different groups of students have different learning styles. Meanwhile, every teacher is unique and each classroom scenario is constantly changing. So educators are looking at hard data and seeking new solutions to help students improve their learning skills. In doing so, students will hopefully excel in grade school and high school, select higher education, choose their college majors, graduate and successfully push forward into their career lives. Computer-aided instructional materials are part of the solution teachers are searching. This is the use of computer software to enhance teaching experience.

At this point, the researcher deemed it timely to develop and evaluate a computer-aided instructional material that would assist both the teachers and students in learning different concepts and skills.

Thus, the importance of the present study can be of benefits to the following: 
TLE Teachers. The material will ease the burden in preparation of plans and materials IT minimizes teachers' load explaining concepts and information, as well as reinforcing the skill.

Parents. This study may help parents realize their children's educational needs, produce support and acceptance of their child's abilities, capabilities and weakness as well.

Students. It enhances students' learning in demonstrable ways. It supplements drills, exercises and simulation activities to reinforce skills concept.

Future Researchers. Discovering further the value and benefits of the use of computers in the classroom setting may be utilized in considering other discipline and bigger population.

The present study is founded on the theory of blended learning (Jacob, 2011). It is a formal education program in which a student learns at least in part through delivery of content and instruction via digital and online media with some element of student control over time, place, path, or pace. Blended Learning can generally be classified into six models, namely: Face to face driver - where the teacher drives the instruction and augments with digital tools; Rotation - students cycle through a schedule of independent online study and face-to-face classroom time; Flex - Most of the curriculum is delivered via a digital platform and teachers are available for face-to-face consultation and support; Labs - All of the curriculum is delivered via a digital platform but in a consistent physical location. Students usually take traditional classes in this model as well; Self-Blend Students choose to augment their traditional learning with online course work; and Online Driver - All curriculum and teaching is delivered via a digital platform and face-to-face meetings are scheduled or made available if necessary. The present study utilized the later model where the students are exposed to digital technology while conducting a face-to-face discussion, by utilizing multimedia elements that includes graphics, sounds, animations, video, and pictures enhancing presentation of lessons involving more learning channels.

This study seeks to design, develop and evaluate Computer-Aided Instructional Material in Teaching Cookery in Grade 10: It's Effectiveness.

Specifically, it sought to answer the following sub-problems:

1. What is the level of performance of the control and experimental group before the study?

2. What is the significant difference on the performance level of the control and experimental group after the study?

3. What is the significant effect of computer aided instructional materials in the level of performance of the students in the experimental group? 


\section{The Conceptual Framework of the Study}

The present study is founded on the theory of blended learning (Jacob, 2011). It is a formal education program in which a student learns at least in part through delivery of content and instruction via digital and online media with some element of student control over time, place, path, or pace. Blended Learning can generally be classified into six models, namely: Face to face driver - where the teacher drives the instruction and augments with digital tools; Rotation - students cycle through a schedule of independent online study and face-to-face classroom time; Flex - Most of the curriculum is delivered via a digital platform and teachers are available for face-to-face consultation and support; Labs - All of the curriculum is delivered via a digital platform but in a consistent physical location. Students usually take traditional classes in this model as well; Self-Blend Students choose to augment their traditional learning with online course work; and Online Driver - All curriculum and teaching is delivered via a digital platform and face-to-face meetings are scheduled or made available if necessary. The present study utilized the later model where the students are exposed to digital technology while conducting a face-to-face discussion, by utilizing multimedia elements that includes graphics, sounds, animations, video, and pictures enhancing presentation of lessons involving more learning channels. The researcher limits this study on the design, development, and evaluation of a computer aided instructional materials material in Cookery. Topics that were included in the material were based on the existing course outline provided by the division. The design of the materials was done using appropriate presentation software available in the local market.

Related literature, studies, and research articles that are relevant in the formation of this research work were reviewed. These studies, journals and literatures established the foundation of this research work. It provided the insights in the conduct of this research.

There is lingering issue on how educational technology integrated in the teaching learning process. This is due to the fact that the mere use of the computers does not mean technology has already integrated in the instruction (Lucido, 2007). Example of this is, students just playing around the internet or playing in the games internet like DOTA and crazy cart. This degrade the technology in the lower standard, giving it a thought that computer is just for games and nothing else.

On the other hand of that thing, through progressive state policies that support technology-in-education, and other new development in the pedagogical practices, our educators today have become more aware and active on adopting state-of-the art educational technology practices that they can possibly adopt. Furthermore, recent changes have also occurred in the area of pedagogical theory and practice. It is now accepted that the 
contribution of the computer to pedagogy makes up for "good instruction" (Lucido, 2007).

But this doesn't mean taking the role of the teachers in the teaching learning process. One assumption is the change of the role of the teacher from the "dispenser of knowledge" to "facilitator of learning". CAI can also help the students in their work. In secondary and tertiary schools, most students use tool applications like word processing, spreadsheets and presentation (Grabe\&Grabe, 2006).

Under CHED memorandum order, series of 2007, it is stipulated that it must improve the classroom teachers' efficacy producing innovative and creative instructional programs or materials that will improve the teachinglearning process. The development of these materials is timely because even without the presence of the teacher, learning takes place.

As Subong (2012) explained, instructional objectives serve as goals that teachers have set in the achievement of a greater goal. They also tell students what is expected of them. Instructional objectives make definite the direction in which teaching leads and become the focus of instruction, not only for the teachers, but also for the students. Without instructional objectives teaching is comparable to a fallen leaf whose destination is dependent on the will of the wind. Without instructional objectives, teachers will have nothing to follow in order to achieve what it should achieve.

He also believed that assessment plays an indispensable role in the educative process. It determines whether the teacher has achieved the goals he set or not. The instructional objectives provide teachers the idea of what is the best assessment tool to be used in a given situation. Moreover, instructional objectives determine the behavior to be measured. Without instructional objectives, the teacher will have no indicator to measure achievement. What then is there to measure anyway with the absence of bases for assessment?

Adelman (2006) stated that many schools struggling to find creative, effective, and feasible approaches to enhance academic achievement and compensate for the number of out-of-field teachers are turning to advances in computer-aided instruction. Although computer accessibility has increased dramatically over the past decade, the evidence on the effectiveness of technology on student achievement has been mixed at best. Determining the potential benefits of computer-aided instruction programs could have significant implications both in addressing the over-whelming need to improve stagnantly low levels of academic achievement and in identifying practical, cost-effective solutions for schools.

Principals may assign students they think would benefit from computerized instruction to the computer labs, resulting in upward biased estimates; and if teachers who are assigned to the lab are those who are more willing to use computerized instruction, the estimated effects could be 
capturing the effectiveness of the teacher rather than the effectiveness of the computer program.

According to Brinton (2005), teachers are required to implement the adopted content standards and to make the connection between standardsbased curriculum and the planning and designing of lessons to ensure that students meet expected content standards. Learners should be exposed to a considerable amount of information through stimulating content. Learners explore interesting content activities. Information is reiterated by strategically delivering information at right time and situation compelling the students to learn out of passion. Eventually, greater flexibility and adaptability in the curriculum can be deployed as per the student's interest.

Barrow, Markman, and Rouse (2008), examines the effect of a popular pre-algebra/algebra computer-aided instruction program, I Can Learn $\odot$, in three US urban schools districts suffering similar problems of underachievement and teacher recruitment. Each school district agreed to the implementation of a within-school random assignment design at the classroom level, thereby avoiding the sources of student and teacher bias previously described. Additionally, since I Can Learn $\odot$ subject lessons are designed so each student progresses through the material at her own pace and the teacher's primary role is to provide targeted help when needed, it is a well-suited program for testing the individualized instruction hypothesis mentioned above.

One of the many obstacles faced in addressing the education system is the combination of limited financial resources and limited conclusive research on which policies are effective in increasing achievement. While schools are always restricted by limited funds, current economic conditions and the potential for additional budget cuts highlight the importance of thoroughly examining the costs involved in implementing any measure of reform. In the case of computer-aided instruction programs, taking all expenses into consideration, the annual cost per lab is nearly $\$ 53,000$. But given the evidence that computer-aided instruction aids achievement by increasing the amount of individual instruction time a student receives, it is possible computer-aided instruction could serve as a substitute for reducing class sizes. When Ingersoll (2008) benchmark the cost of computer-aided instruction against compensation costs associated with increasing the teaching staff, they conclude the two are equally cost-effective. This is a significant result as it suggests an alternative, and likely easier, means of increasing instruction time for urban and rural districts that struggle to hire highly qualified teachers.

Computer-Aided Instructional Material brings with several potential benefits as a teaching/learning medium. These include self-paced learning, self-directed learning, the exercising of various senses and the ability to represent content in a variety of media. With self-paced learning, learners can move as slowly or as quickly as they like through the program. If they 
want to repeat some task or review some material again, they can do so as many times as they choose. The program will not tire or complain about repetitions. Learners can skip over a topic if information is already known, making the learning process more efficient. With self-directed learning, learners can decide what they want to learn and in what order. Learners have different learning styles and use different learning strategies (Lee, Grigg, and Dion, 2007).

When learners can learn in a way that suits them, improvements in the effectiveness of learning process normally ensure. Humans are multisensory animals. The more senses through which we receive information, the easier it is to remember. According to Wenglinsky (2008) people remember $20 \%$ of what they hear, $40 \%$ of what they see and hear and $75 \%$ of what they hear, see and do. The fact that computers can present information in variety of ways, then there is a big possibility that it can enhance the learning process.

The major purpose of the study of Alimondo (2005) is to determine the effectiveness of computer-assisted instruction in Basic English in the University of Baguio. The following problems were investigated: (1) what are the performance levels of the experimental group (treated with CAI) and the control group (treated with lecture method) in the pretest and posttest along general vocabulary, parts of speech, embedding, and writing mechanics. (2) Are there significant differences in the performance levels identified aspects in the pretest and posttest? (3) Is there a significant difference on the performance levels between the experimental group and the control group along the four identified aspects? (4) What is the level of correlation of the performance of both groups?

She found out that the students in English 101 control group and experimental group showed a poor level of performance along general vocabulary, parts of speech, embedding, and writing mechanics. However, there was an increase in students' performance in the experimental group than the students in the control group.

It is further found out that students performed better with the use of computer-assisted instruction than in the use of lecture method. The students' performance level in the control group is the same along embedding and writing mechanics whereas different in general vocabulary and parts of speech. On the other hand, the students' performance levels in the experimental group is the same along general vocabulary and parts of speech whereas different in embedding and writing mechanics. The performance levels of students in Basic English vary in favor of computer assisted instruction along general vocabulary, parts of speech, embedding and writing mechanics. There is a very high overall correlation in the performance levels of students in Basic English along general vocabulary, parts of speech, embedding, and writing mechanics with computer assisted 
instruction while marked correlation in the students' overall performance levels in the aforementioned subject areas in the lecture method.

The objective of Ragasa's (2008) study is to determine if there is a significant difference in the effects of the treatment and control groups on achievement as well as on attitude as measured by the posttest. A class of 38 sophomore college students in the basic statistics taught with the use of computer-assisted instruction and another class of 15 students with the use of the traditional method from the University of the East, Manila (SY 20072008) were the focus of this study. The research method used was the quasiexperimental, nonequivalent control group design. The statistical tool was the Multiple Analysis of Covariance. The researcher made use of the CDROM prepared by Math Advantage to serve as the teaching medium for the experimental group. The following summarizes the findings of the study. The achievement posttest of the treatment group has higher estimated marginal means than the control group and it is reversed in the attitude posttest. Using Hotelling's Trace for the multivariate test, the achievement pretest, attitude pretest, and the two groups.

The study of Huxford (2009), examines the relative efficacy of computer assisted instruction (CAI). A review of literature is examined with regard to the positive and negative aspects of the use of CAI in the university classroom. An experimental design was outlined incorporating the use of 3 teachers instructing 3 separate sections of volunteer students on a topic. The design measured the differences between affective learning, cognitive learning and student perceptions of teacher nonverbal immediacy. Participants were randomly assigned to a class, which utilized either: lecture, handout or the use of CAI. Results showed significant differences between teachers and a slight difference in perceived immediacy in instruction. The findings suggest that the use of CAI may not be as useful in the instructional context as was previously believed. He found out that the matter of external validity must be addressed with regard to this study. External validity consists of a great many variables. Of importance in this study are: history, testing, selection, and instrumentation. The testing variable may have been confounded because the students lacked reinforcement for performing well on the quiz. Specifically, although the students were informed that their grades would be in no way changed by the quiz they would take, some students may have, for this very reason, not taken the quiz seriously. If they could have been lead to believe the amount of extra credit was contingent on the quiz scores, the cognitive assessment may have increased.

Previous research had mixed suggests in using CAI for more effective teaching. This study seems to concur with previous findings, yet in a more specific way with regards to a decrease in immediacy and no effects in affective learning and cognitive learning. Through experimental design, this study evaluated the ways in which teachers perform their functions, 
whether it be through the use of handouts, straight lecture, or the use of CAI. Future research should examine the ways in which teachers understand and use CAI. The differences between teachers in this study were not significant enough for statistical measures, yet there were differences between the teachers. In this study, the teachers did not have the opportunity to examine the use of CAI before their designated instruction period. This may have confounded the ways in which they may have used the medium. Specifically, the insignificant finding which stated that Teacher had more affective learning using CAI than in other conditions brings a question of computer experience. This teacher had a great deal of experience with computers and was comfortable with their workings, although he also did not have any experience with the CAI presentation before hand. Of importance to future research should be the familiarity of computer use within the teacher.

In this study, there were observable differences in the ways which teachers used the CAI medium. Specifically, they seemed tied to the computer terminal during their presentation of the Toulmin Model. This would certainly have effects on perceptions of immediacy and possibly on perceptions of affective learning as well. Perhaps with the addition of a remote for the computer, perceptions of immediacy may change.

The purpose of the research of Warren (2012) was to investigate the effects of including adaptive confidence strategies in instructionally sound computer-assisted instruction (CAI) on learning and learner confidence. Seventy-one general educational development (GED) learners recruited from various GED learning centers at community colleges in the southeast United States were randomly assigned to one of three levels of independent variable: Absence of Adaptive Confidence Strategies Condition (nonadaptive, or NA), Adaptive Confidence Strategies with Program Control Condition (PC), or Adaptive Confidence Strategies with Shared Control Condition (SC). In the NA condition, learners received instructionally sound CAI that did not measure nor respond to changes in learner confidence during the learning process. In the other two conditions, learners received instructionally sound CAI that did measure and respond to changes in learner confidence by implementing various confidence building and confidence sustaining strategies during the learning process, but that differed in the amount of control allowed to learners.

Implementing the adaptive confidence strategies involved the CAI program collecting learner performance data (e.g., from practice items) and learner self-reported confidence data (from an embedded confidence analysis), interpreting the data using a confidence diagnosis-prescription rubric in order to diagnose the confidence state of the learner, and finally prescribing confidence strategies based on the diagnosis. The two dependent variables were learning and learner confidence. Learning was measured using a posttest consisting of eight items that reflected the content taught in 
the CAI. Learner confidence was measured on the exit survey using a shortened form of the IMMS. Both learning and learner confidence were expected to be greater for CAI containing adaptive confidence strategies than for CAI not containing adaptive confidence strategies. In addition, this study explored the effect that the extent of control (i.e., choice of learning task and branching) provided to learners in the form of an adaptive confidence strategy had on learning and learner confidence.

\section{METHODS}

The study employed conceptual paradigm which is the InputProcess-Output (IPO) Model of the system approach.

Input which includes, course outline, references, supplies and materials and technical manuals like computer units, peripherals and accessories. The pretest and posttest that was given to the respondents.

Output which is the design and development of computer-aided instructional materials in cookery. The administration of pretest and posttest, statistical treatment of data and analysis and interpretation of gathered data.

Output, is the effectiveness of computer aided instructional materials in teaching cookery in grade 10 students.

This study seeks to design, develop and evaluate Computer-Aided Instructional Material in Teaching Cookery in Grade 10: It's Effectiveness. Specifically, it sought to answer the following sub-problems:

What is the level of performance of the control and experimental group before the study?

What is the significant difference on the performance level of the control and experimental group after the study?

What is the significant effect of computer aided instructional materials in the level of performance of the students in the experimental group?

The study was guided by the following hypotheses:

There is no significant difference between the performance level of the control group and experimental group in their pre and posttest.

There is no significant effect of the proposed computer aided instructional materials in Cookery in the experimental group.

In this study, experimental method of research was used, the pretest and posttest design. The control group and the experimental group were both given a pretest, then exposed to different teaching methods. The experimental group was exposed to computer aided instructional materials while the control group was taught using the traditional approach. Both groups were given a posttest.

The respondents of this study were taken from two heterogeneous sections of Grade 10 students. Lottery was used as sampling technique in selecting the control and experimental group. 
This study utilizes the pre-test and posttest as its main instrument. The tests were developed by the researcher for the said purpose. Fifty items for each test was allotted based on the table of specification and curriculum guide grade 10 . Both test had the same difficulty to ensure the effectiveness of the treatment to be imposed in the experiment.

1. Percentage. This was used as descriptive statistics or something that describes a part of the whole.

$\mathrm{P}=\underline{\text { no. of correct response }}$

No. of cases

2. T-test. This was used to measure the degree of difference between the pre and posttest mean scores of the respondents.

The following formula helped compute the T-test

The Mean

Formula:

$X=\frac{\sum}{N}$

$\sum=$ the arithmetic

$X \sum=$ sum of scores

$\mathrm{N}=$ number of scores

Sub-problem no. 1

\section{RESULTS}

What is the level of performance of the control and experimental group before the study?

The Mean Percentage Score of the control group before the study was $35 \%$ while the experimental group was $34 \%$. It revealed that the control group and the experimental group was almost the same in terms of performance level.

Sub-problem no. 2

What is the significant difference on the performance level of the control and experimental group after the study?

The computed value of the control and experimental group was 45 which is higher than the critical value of 2 . This means that there is a significant difference between the performance levels of the two groups. Therefore reject the null hypotheses.

Sub-problem no. 3

What is the significant effect of computer aided instructional materials in the level of performance of the students in the experimental group? 
The Percentage score of the experimental group before the study was $34 \%$ and goes up to $97 \%$. It showed that there is a significant effect of computeraided instructional materials in the performance level of the students.

\section{CONCLUSIONS}

Based on the findings following conclusions was drawn:

That students who used computer aided instructional materials performed wellin class and can interact with their classmates actively.

The result of the experimental group increases in the post test, which signifies that learning is more fun and took place using the computer aided instructional materials.

Computer aided instruction enhances the learning abilities of the students, hence students will appreciate importance of technologies which will lead to a more meaningful learning interactions.

\section{REFERENCES}

Adelman, C. (2006). The Toolbox Revisited:Paths to Degree Completion from high School through college. us depaRTMENT OF EDUCATION, WASHINGTON D.C.

Alimondo, J. (2005). Effectiveness of Computer-Assisted Instruction in Basic English in the University of Baguio (Master's Thesis). Saint Louis, Baguio City.

Arcilla, M. (n.d.). E-Sci : An Intelligent Computer Aided Instruction. Academic Paper Review. Caloocan City: AMAM University.

Barlis, J. ,. (2013). Effectiveness of Simulation and Computer Assisted Instruction (CAI) on the Performance of Students under Regimental Training on Selected Topics in Physics II. International Journal of Applied Physics and Mathematics, Vol. 3, No. 1.

Barrow, L. M. (2008). Technology's Edge: The Educational Benefits of Computer -Aided Instruction. National Bereau of Economic Research Working Paper, 14240.

Brinton, E. (2007). Electronic Education System Model. Computers \& Education,, vol. 36, 43-44.

Britannica Encyclopedia. (2011).

Cohen, L. ,. (2007). Research Methods in Education. London: Routledge.

Fraenkle, J. R., \& and Hyun, H. H. (2012). How tro design and evaluate research in education, 8th edition. New York: McGraw-Hill Education (Asia).

Huxford, D. E. (2009). the Relative Efficacy of Computer Assisted Instruction (Thesis). Eberly College of Arts and Sciences West Virginia University. 
Lee, J. G. (n.d.). The Nation's Report Card: Mathematics 2007.

Oguz, S. (2011). The Effects of the Computer-Based Instruction on the Achievement and Problem Solving Skills of the Science and Technology Students (Master's Thesis). Cyprus International University.

Pilli, O. (2007). The Effects of Computer-Assisted Instructionon the Achievement, Attitude, Retention of Fourth Grade Mathematics Course. (Dissertation). Social Sciences of Middle East Technical University.

Ragasa, C. (2008). A Comparison of Computer Assisted Instruction and Traditional Method of Teaching Basic Statistics. Journal of Statistics Education, Volume 16, Number 1.

Spradlin, K. (2012). The Effectiveness of Computer-Assited Instruction in Developmental Mathematics (Dissertation). Liberty University.

Subong, R. C. (2012, October). A Study on e-Learning for Philippines. International Journal of Multimedia and Ubiquitous Engineering, Vol. 2 No. 4,.

Warren, R. D. (2012). the effect of Adaptive Confidence Strategies in Computer-Assisted Instruction on Learning and Learner Confidence (Dissertation) . The Florida University.

Wenglinsky, H. (2009). Does it Compute? The Relationship Between Educational Technology and Student Achievement in Mathematics. ERIC Document Reproduction Service No. ED425191. 